\title{
EXAME CITOPATOLÓGICO DE COLO UTERINO Subsídios Para Intervenção Na Atenção Primária
}

\author{
Aline Raquel Ceolin 1 \\ Arlete Regina Roma ${ }^{2}$ \\ Teila Ceolin ${ }^{3}$ \\ Patrícia Tuerlinckx Noguez ${ }^{4}$
}

\section{RESUMO}

Objetivo: analisar resultados de exames citopatológicos de colo uterino de mulheres assistidas pela Estratégia de Saúde da Família de um município da região Sul do Brasil. Métodos: abordagem quantitativa, descritiva, de fonte documental. Os dados foram coletados entre 2008 e 2010, no livro de registro, e analisados partir do agrupamento dos mesmos e da distribuição de frequência. Resultados: foram avaliados 774 registros de exames citopatológicos, com predominância da faixa etária entre 30 e 39 anos. Entre os que apresentaram corrimentos vaginais, houve prevalência de Gardnerella vaginalis. Apenas três exames foram compatíveis com Lesão Intra Epitelial de Baixo Grau. Conclusões: os resultados desse estudo contribuem para fornecer indicadores para subsidiar ações de educação em saúde para as mulheres atendidas no serviço.

Palavras-chave: Saúde da mulher. Prevenção de câncer de colo uterino. Neoplasias uterinas. Estratégia de Saúde da Família.

\section{PAP TEST OF CERVIX SUBSIDIES FOR INTERVENTION IN PRIMARY HEALTH}

\begin{abstract}
Objective: to analyze results of pap exams of cervix from women attended in a Family Health Strategy (FHS) from a city in the southern region of Brazil. Methods: research with quantitative and descriptive approach, with a documental source. Data collection occurred between 2008 and 2010, through the book of register; analysis of data occurred through grouping the same and the distribution of frequency. Results: were evaluated 774 records of pap exams, with predominance of age between 30 and 39 years old. Among the ones who presented vaginal discharge, there was prevalence of Gardnerella vaginalis. Only three exams were compatible with Low Grade Intraepithelial Lesion. Conclusion: the results of this study contribute in the sense of provide indicators about the pap exam to, in this way, subsidize actions of education in health to attended women in service.
\end{abstract}

Keywords: Women's health. Cervix neoplasms prevention. Uterine neoplasms. Family health strategy.

\footnotetext{
Enfermeira. Especialista em Saúde da Família pela Universidade Federal de Pelotas (UFPel). alineraquelceolin@hotmail.com

Mestre em Enfermagem. Professora do Curso de Enfermagem (Unijuí). arleter@unijui.edu.br

3 Doutora em Ciências. Professora da Faculdade de Enfermagem da UFPel. teila.ceolin@gmail.com

4 Doutoranda do Programa de Pós-Graduação em Enfermagem (UFPel). Professora da Faculdade de Enfermagem (UFPel). patriciatuer@hotmail.com
} 
O câncer de colo de útero é um problema de saúde pública, que atinge todas as camadas sociais e regiões geoeconômicas. Em 2012 ocorreram 527 mil casos novos estimados no mundo, os quais foram responsáveis pelo óbito de 265 mil mulheres. É o quarto tipo de câncer mais comum entre as mulheres. Sua incidência é cerca de duas vezes maior em países menos desenvolvidos quando comparada aos países mais ricos (Brasil, 2014a).

Segundo o Instituto Nacional do Câncer (Inca-BR), o câncer de colo do útero, quando diagnosticado como localização primária do tumor, é a quarta causa de morte por câncer entre as mulheres no país, mantendo esta posição entre os anos de 2002 e 2011. No Brasil, em 2014, foram estimados 15.590 casos novos de câncer do colo do útero (Brasil, 2014a).

O controle desta neoplasia obedece à estratégia de prevenção secundária baseada na citologia cervical. Esta técnica de detecção, conhecida popularmente como Papanicolaou ou simplesmente exame preventivo, vem sendo realizada há mais de 30 anos. Vários estudos realizados apontam a prevalência do câncer de colo uterino em diferentes Estados brasileiros e países (Arzuaga-Salazar; de Souza; de Azevedo-Lima, 2012; Borges; Dotto; Koifman et al., 2012; Mendoza et al., 2013).

A literatura aponta que existe íntima relação entre o câncer de colo de útero, o comportamento sexual das mulheres e a transmissão de agentes infecciosos, revelando-se o principal fator de risco para o câncer do colo do útero a infecção pelo papilomavírus humano (HPV), o qual, isoladamente, não representa causa suficiente para o surgimento dessa neoplasia. Outros fatores como a imunidade, a genética e o comportamento sexual parecem influenciar na regressão ou na persistência da infecção, bem como na progressão para lesões precursoras ou câncer. Além disso, a idade acima de 30 anos e o tabagismo elevam o risco para o desenvolvimento do câncer do colo do útero (Brasil, 2014a).

Outro fator de risco de grande significância é a história de Doenças Sexualmente Transmissíveis (DSTs), principalmente na exposição ao vírus papiloma humano (HPV). Sabe-se que o surgimento do câncer do colo do útero possui importante asso- ciação à infecção por um dos 13 tipos oncogênicos de HPV (Brasil, 2014a). O vírus HPV é responsável por $95 \%$ dos casos de câncer de colo do útero, apresentando a segunda maior taxa de incidência entre os cânceres que atingem as mulheres, atrás apenas do de mama (Brasil, 2011a). Estima-se uma redução de até $80 \%$ na mortalidade por este tipo de câncer a partir do rastreamento de mulheres na faixa etária de 25 a 64 anos com o exame de Papanicolaou e tratamento das lesões precursoras com alto potencial de malignidade ou carcinoma in situ. Para tanto, é necessário garantir a organização, a integralidade e a qualidade do programa de rastreamento, bem como do tratamento das pacientes (Brasil, 2014b).

Diante disso, os profissionais devem ter consciência, no ato do exame, que cada pessoa tem sua própria percepção sobre os procedimentos que envolvem a prevenção do câncer cérvico-uterino. Assim, o enfermeiro pode ser considerado um pilar no processo educativo-dialógico, por propiciar informações e empoderar a comunidade visando à adoção de comportamentos mais saudáveis (Melo et al., 2012). Quando as mulheres recebem adequadas informações tornam-se mais conscientes e buscam realizar ações de prevenção e consequentemente diagnóstico precoce dos problemas de saúde, incluindo a prevenção do câncer de colo de útero, que é detectado com a realização da coleta do citopatológico cervical (Shiavon, 2009).

Nessa ótica, esta pesquisa teve como objetivo analisar resultados de exames citopatológicos de colo uterino de mulheres assistidas pela Estratégia de Saúde da Família (ESF) de um município da Região Sul do Brasil.

\section{MATERIAIS E MÉTODOS}

Este artigo faz parte da pesquisa " $\mathrm{O}$ que revela o citopatológico de colo uterino de uma Unidade de Saúde da Família em um município da Região Noroeste - RS", a qual se caracterizou por ser uma retrospectiva com abordagem quantitativa, descritiva, de fonte documental, na qual foram avaliados os resultados dos exames citopatológicos de colo 
uterino de mulheres assistidas em uma Unidade Básica de Saúde (UBS) com equipe de Estratégia de Saúde da Família (ESF) de um município da Região Noroeste do Rio Grande do Sul, Brasil.

O referido município tem uma população de 13.970 habitantes e uma equipe de ESF, a qual atua em uma área na qual residem 4.300 pessoas, abrangendo quatro bairros e sete comunidades da área rural (Brasil, 2011b). Optou-se por investigar esta equipe de ESF, devido ao fato de esta trabalhar com uma população delimitada, o que permite o monitoramento periódico das mulheres.

Os dados foram coletados no livro de registros para exames citopatológicos do colo uterino da UBS. O período estudado compreendeu os anos 2008 a 2010, definidos porque se entende que três anos permitem a análise a partir dos objetivos propostos.

Neste estudo observou-se preceitos da Resolução no 196/96 (Brasil, 1996) do Conselho Nacional de Saúde, que trata da pesquisa envolvendo seres humanos, bem como o Capítulo III da Resolução do Conselho Federal de Enfermagem (Cofen) 311/2007, do Código de Ética dos Profissionais de Enfermagem que trata do ensino, da pesquisa e da produção técnico-científica.

O projeto foi aprovado pelo Comitê de Ética em Pesquisa da Universidade Regional do Noroeste do Estado do Rio Grande do Sul - Unijuí, sob o parecer $n^{\circ} 193.0 / 2011$.

Para coleta de dados foi utilizado um quadro com as seguintes informações: número da lâmina, data da coleta do exame, resultados de citologia e flora vaginal e idade. Após a coleta os dados foram analisados e os resultados encontrados foram comparados com os referenciais teóricos e outros estudos científicos.

\section{RESULTADOS}

O presente estudo avaliou os registros de 774 exames citopatológicos cervicais, de uma equipe de ESF, de um município da Região Noroeste do Rio Grande do Sul, entre 2008 e 2010 (Figura 1).
Figura 1 - Distribuição das mulheres de acordo com a faixa etária e ano das coletas de exame de Papanicolau. Rio Grande do Sul, Brasil, 2011

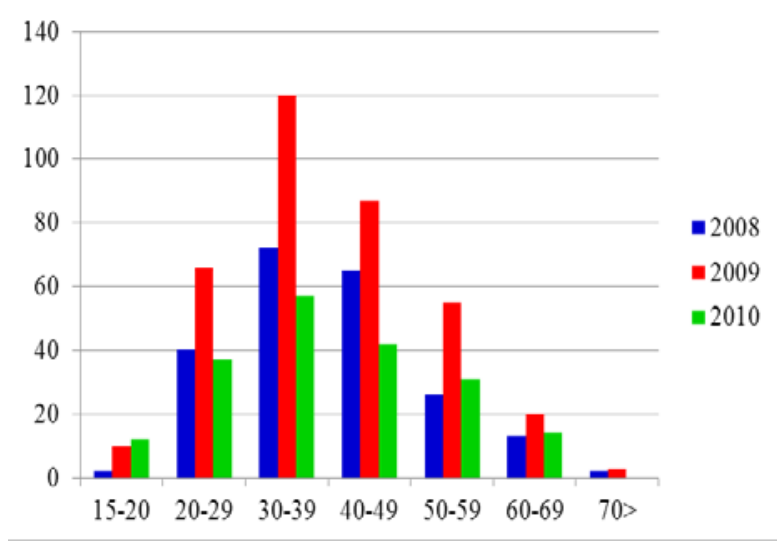

Fonte: Livro de registros dos exames citopatológicos do colo uterino da UBS investigada.

De acordo com a Figura 1, a faixa etária predominante foi entre 30 e 39 anos, representando um total de 249 mulheres $(32,17 \%)$ das coletas entre 2008 e 2010. Somando-se a faixa etária de 40 a 49 anos obtém-se um percentual de 57,23\%.

Em 2009, no município investigado, foram realizados mais exames em comparação aos outros anos. Isso ocorreu devido à realização de uma campanha local, de Prevenção do Câncer de Colo do Útero, na qual foram mobilizados profissionais da saúde para que fizessem uma conscientização da importância da realização do citopatológico cervical.

Os dados também revelam que a cobertura das mulheres com menos de 20 anos é baixa, totalizando 24 mulheres (3,10\%). 57,23\% das coletas de exame de Papanicolaou foram realizadas na faixa etária corresponde a 30 a 49 anos, corroborando informações obtidas na literatura.

No presente estudo houve três resultados (0,38\%) compatíveis com Lesão Intraepitelial de Baixo Grau (NIC I) e nenhum para lesão intraepitelial de alto grau (NIC III), o que mostra a importância da realização rotineira do exame, com vista à detecção precoce.

Nos três anos analisados nesta pesquisa apenas uma mulher foi diagnosticada com Chlamydia trachomatis (Figura 2), a qual é um importante agente 
causador de endocervicite em mulheres sexualmente ativas, podendo ser assintomática, ou apresentar sintomas inespecíficos.

Figura 2 - Resultados do exame Papanicolau. Rio Grande do Sul, Brasil, 2011

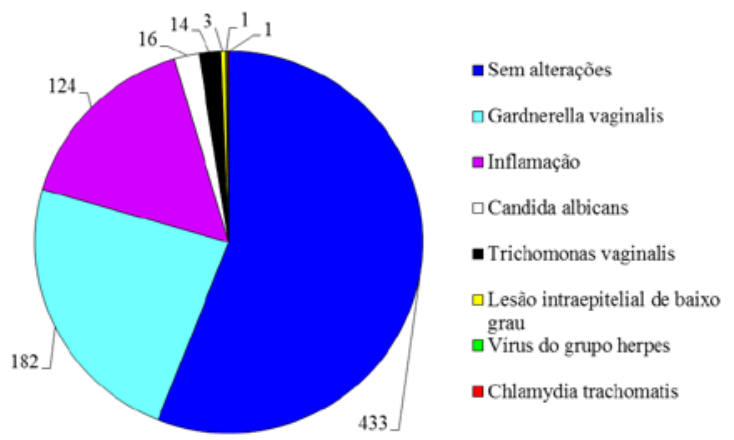

Fonte: Livro de registros dos exames citopatológicos do colo uterino da UBS investigada.

\section{DISCUSSÃO}

De acordo com dados do Inca (Brasil, 2011a), a incidência do câncer do colo do útero em mulheres até 24 anos é muito baixa, a maioria dos casos é diagnosticada no estágio I e o rastreamento é menos eficiente para detectá-los.

A baixa cobertura encontrada neste estudo em mulheres com menos de 20 anos $(3,10 \%)$ está de acordo com a justificativa do Inca, em relação à não antecipação do início do rastreamento dos 25 para os 20 anos, tendo em vista o impacto muito limitado e avaliado como não vantajoso (Brasil, 2011b). Há nessecidade, no entanto, de ações de orientação em saúde para estas muheres adolescentes, tendo em vista o início cada vez mais cedo da vida sexual e a consequente exposição ao HPV. Cabe ao enfermeiro buscar estratégias para sensibilizar o público jovem com a intenção de rastrear e, se necessário, tratar precocemente alguma neoplasia.

Em 2014 o Ministério da Saúde iniciou a vacinação gratuita contra o HPV para o grupo de 11 a 13 anos e, em 2015, para as adolescentes de 9 a 11 anos. A vacina que está sendo utilizada é a quadrivalente, usada na prevenção contra quatro tipos de $\operatorname{HPV}(6,11,16$ e 18), uma vez que dois deles (16 e 18) respondem por $70 \%$ dos casos de câncer. A meta do Ministério da Saúde é vacinar $80 \%$ do público-alvo, de aproximadamente 5,2 milhões de pessoas (Brasil, 2014b).

O esquema de vacinação segue a recomendação da Organização Pan-Americana de Saúde (Opas), com três doses (dose inicial; segunda dose seis meses após e a terceira dose deverá ser aplicada cinco anos após a primeira). Um dos benefícios do esquema vacinal estendido é que a terceira dose, cinco anos depois, funciona como um reforço, prolongando o efeito protetor contra a doença. Este esquema vacinal já é utilizado em países como Canadá, México, Colômbia, Chile e Suíça (Brasil, 2014b).

A incidência do câncer do colo do útero intensifica-se a partir dos 30 anos, aumentando seu risco rapidamente até atingir o pico etário entre 50 e 60 anos. Com exceção do câncer da pele não melanoma, esse tumor é o que apresenta maior potencial de diagnóstico prévio e cura quando diagnosticado precocemente (Brasil, 2014a).

O principal fator de risco para o desenvolvimento de lesões intraepiteliais de alto grau e do câncer do colo do útero é a infecção pelo papilomavírus humano (HPV). Existem hoje 13 tipos de HPV reconhecidos como oncogênicos pela Agência Internacional para Pesquisa sobre o Câncer (Iarc). Desses, os mais comuns são o HPV16 e o HPV18, responsáveis por aproximadamente $70 \%$ dos casos (Brasil, 2014a).

Segundo recomendação do Ministério da Saúde Brasil (Brasil, 2011b), as mulheres dos 25 aos 60 anos de idade, ou as que iniciaram sua atividade sexual anteriormente a esta faixa etária, devem fazer o exame uma vez por ano e, após dois exames anuais consecutivos negativos, devem realizar a cada três anos.

De acordo com os dados do Sistema de Informação da Atenção Básica (Siab) de 2010, do município investigado, o total de mulheres residentes na área de abrangência da equipe da ESF pesquisada era de 1.184 mulheres, em uma faixa etária que compreendia entre 15 e maiores de 60 anos, com variação de 1.156 em 2009 e 1.105 em 2008 
(Brasil, 2011a). Ao identificar a média entre os três anos, encontra-se $22,46 \%$ das mulheres desta faixa etária com a realização da coleta do citopatológico.

Uma pesquisa (Soares; Silva, 2010) realizada na rede pública municipal de saúde de Igarapava-SP, identificou 1.501 coletas de exame de Papanicolaou, apresentando uma cobertura de $14,9 \%$ da população feminina na faixa etária de 25 a 59 anos, com a faixa etária predominante das coletas de 20 a $34 \operatorname{anos}(43,2 \%)$.

Em outro estudo realizado (Batista et al., 2012) em Goiânia-GO, com 15.615 mulheres que se submeteram ao exame citopatológico do colo do útero nas Unidades Básicas de Saúde, entre 2006 e 2008, a maioria $(57,6 \%)$ encontrava-se na faixa etária de 20 a 39 anos e $19,3 \%$ entre 40 e 49 anos de idade. Já no estudo (Leite et al., 2010) realizado com 277 mulheres, percebeu-se que quase metade delas tinha de 25 a 34 anos (49,5\%), seguidas daquelas com 35 anos ou mais, as quais totalizaram 99 mulheres $(35,7 \%)$. As mais jovens (18 a 24 anos) corresponderam a $14,8 \%$ do total.

Em relação às DSTs, um estudo (Lessa et al., 2012) desenvolvido no Ceará, com mulheres privadas de liberdade, observou-se a presença, nas colonizações cérvico-vaginais, de Gardnerella/ mobiluncus, com 21,8\%, seguidos de Trichomonas vaginalis, $12 \%$, e Candida sp. em 5,8\%. As frequências de amostras com atipias celulares mostraram índices de 4,1\% para atipias de significado indeterminado, lesões intraepiteliais de baixo grau 3,2\% e lesões intraepiteliais de alto grau 2,5\%.

Quanto às lesões intraepiteliais, na NIC I, as alterações limitam-se a um terço do epitélio de revestimento da cérvice, sendo classificada como lesão de baixo grau de malignidade (Brasil, 2014c). Aproximadamente $60 \%$ das mulheres com NIC I apresentam regressão espontânea; 30\% mostram persistência da lesão e menos de $10 \%$ evoluem para NIC III. A conduta clínica é acompanhar a mulher e repetir a citologia em seis meses (Brasil, 2011a).

Comparando o tecido neoplásico com o normal, detectou-se maior influência do HPV em mulheres com câncer cervical do que nas sem alterações, le- vando-se em consideração que a prevalência desse vírus aumenta de acordo com a gravidade da lesão. Além disso, mulheres infectadas apresentam risco aumentado de progressão de lesões de baixo grau para de alto grau (Brasil, 2011a).

Um estudo realizado com mulheres residentes em Juiz de Fora (MG) identificou que as neoplasias malignas representaram $17,6 \%$ do total de 1.623 óbitos ocorridos em mulheres em 2006, sendo o câncer de colo do útero a quarta causa de morte (Rodrigues; Bustamante-Teixeira, 2011).

Já no estudo de Oliveira et al. (2008), realizado com objetivo de determinar a frequência de infecção por Chlamydia trachomatis em pacientes com e sem lesões intraepiteliais cervicais, atendidas em ambulatório especializado no Recife-PE, constatou-se a prevalência de infecção por C. trachomatis em pacientes com citologia oncótica normal de 14,3\% e em pacientes com citologia alterada de $80 \%$. O principal fator associado à presença de infecção foi a história pregressa de DST.

A ausência de diagnóstico e tratamento representa um grave problema de saúde pública, uma que as DSTs podem evoluir para sérias complicações como endometrite, doença inflamatória pélvica, esterilidade e infecções neonatais, pulmonares e oftálmicas (Benzaken et al., 2010). Neste sentido, vale destacar a importância da realização de estudos que investiguem as infecções vaginais, pois além de apresentarem sintomas desagradáveis, como prurido, corrimento, disúria, alguns estudos sugerem uma ligação entre infecções genitais e neoplasia cervical, principalmente pelo papilomavírus humano (HPV) (Lessa et al., 2012).

Recomenda-se que todas as Unidades Básica de Saúde estabeleçam, dentro de suas prioridades, um programa que incentive o exame citopatológico, facilitando o acesso às mulheres e organizando seus registros com dados que serão relevantes para futuros estudos, intensificando a procura pelas usuárias para realização da coleta do citopatológico cervical (Risso; Soares, 2011). 


\section{CONCLUSÕES}

Os resultados desse estudo podem contribuir no sentido de fornecer indicadores sobre o exame de prevenção (citopatológico) e identificação dos principais agentes infecciosos. Consequentemente poderá colaborar com a maior cobertura deste exame para o rastreamento do câncer do colo do útero e identificação precoce das lesões precursoras no município estudado.

A partir disso é possível fazer uma reflexão sobre os investimentos que são realizados a fim de sanar este problema, e, na maioria das vezes, aumentam a oferta dos exames citológicos, mas deixam a desejar em relação à melhora da qualidade do atendimento e à realização de práticas educativas. Desta forma, percebe-se que é frequente ocorrer um olhar reducionista acerca desta problemática, além do distanciamento desta prática das ações de promoção da saúde.

O êxito do rastreamento do câncer de colo de útero dependerá, entre outros fatores, da ampliação da cobertura da população feminina com a realização da citologia oncológica; da reorganização da assistência clínico-ginecológica às mulheres nos serviços de saúde; da capacitação dos profissionais de saúde e do estabelecimento de intervenções mais humanizadas e equitativas.

Espera-se que este estudo tenha colaborado no sentido de fornecer indicadores sobre o exame de prevenção (citopatológico), como a prevalência das lesões precursoras e invasivas do colo uterino e identificar os principais agentes específicos. Consequentemente poderá contribuir para a maior cobertura deste exame para o rastreamento do câncer do colo do útero e identificação precoce das lesões precursoras no município estudado.

\section{REFERÊNCIAS}

ARZUAGA-SALAZAR, M. A.; DE SOUZA, M. L.; DE AZEVEDO-LIMA, V. L. El cáncer de cuello de útero: un problema social mundial. Rev Cubana Enfermer, v. 28, n. 1, p. 63-73, 2012.
BATISTA, M. L. S. et al. Resultados citopatológicos de mulheres que realizaram exame do colo do útero em um laboratório escola da Universidade Federal de Goiás, Goiânia-GO: estudo de prevalência. J health sci, v. 30, n. 3, p. 201-205, 2012.

BENZAKEN, A. S. et al. Prevalência da infecção por Clamídia e Gonococo em mulheres atendidas na Clínica de DST da Fundação Alfredo da Matta, Manaus, Amazonas. DST j bras doenças sex transm., v. 3, n. 22, p. 129-134, 2010.

BORGES, M. F. S. O. et al. Prevalência do exame preventivo de câncer do colo do útero em Rio Branco, Acre, Brasil, e fatores associados à não-realização do exame. Cad. saúde pública, v. 28, n. 6, p. 1.156-1.166, 2012.

BRASIL. Ministério da Saúde. Resolução 196/96 sobre pesquisa envolvendo seres humanos. Brasília: Ministério da Saúde, 1996.

Instituto Nacional de Câncer. Coordenação Geral de Ações Estratégicas. Divisão de Apoio à Rede de Atenção Oncológica. Diretrizes brasileiras para o rastreamento do câncer do colo do útero. 2011a. Disponível em: <http://colposcopia.org.br/ files/consensos/6-1144611105.pdf $>$. Acesso em: 28 out. 2014 .

Ministério da Saúde. Sistema de Informação da Atenção Básica (Siab). Cadastramento familiar-Rio Grande do Sul-Santo Augusto. Mulheres entre 15 anos e $>60$ anos. 2008 a 2010. Estratégia de Saúde da Família. 2011b. Disponível em: $<$ http://tabnet.datasus.gov.br/cgi/tabcgi.exe?siab/ cnv/siabfRS.def $>$. Acesso em: 12 out. 2011.

Instituto Nacional de Câncer (BR). Ministério da Saúde amplia faixa etária que vai receber vacina gratuita contra o HPV. 2014a. Disponível em: <http://www2.inca.gov.br/wps/wcm/connect/ agencianoticias/site/home/noticias/2013/ministerio_saude_amplia_faixa_etaria_receber_vacina contra_hpv>. Acesso em: 22 dez. 2011.

Instituto Nacional de Câncer. Estimativa 2014: incidência de Câncer no Brasil. 2014b. Disponível em: $<$ http://www.inca.gov.br/estimativa/2014/estimativa-24042014.pdf>. Acesso em: 22 dez. 2015. 
. Instituto Nacional de Câncer (BR). HPV e câncer - perguntas mais frequentes. 2014c. Disponível em: $<$ http://www1.inca.gov.br/conteudo_ view.asp?id=2687>. Acesso em: 22 dez. 2014.

CONSELHO FEDERAL DE ENFERMAGEM. COFEN. Resolução COFen 311/2007: Aprova a reformulação do Código de Ética dos Profissionais de Enfermagem. COFen, 2007. Disponível em: $<$ http:// se.corens.portalcofen.gov.br/codigo-de-etica-resolucao-cofen-3112007>. Acesso em: 12 dez. 2011.

LEITE, S. R. R. F. et al. Perfil clínico e microbiológico de mulheres com vaginose bacteriana. Rev bras ginecol obstet, v. 32, n. 2, p. 82-87, 2010.

LESSA, P. R. A. et al. Presença de lesões intraepiteliais de alto grau entre mulheres privadas de liberdade: estudo documental. Rev latinoam enferm, v. 20, n. 2, p. 354-361, 2012.

MELO, M. C. S. C. DE et al. O enfermeiro na prevenção do câncer do colo do útero: o cotidiano da atenção primária. Rev. bras. cancerol. v. 58, n. 3, p. 389-398, 2012.

MENDOZA, L. P. et al. Características clínico-demográficas y tipificación del virus de papiloma humano en mujeres paraguayas con citologías negativas para lesión escamosa intraepitelial. Mem. Inst. Investig. Cienc. Salud, v. 10, n. 1, p. 46-55, 2013.

OLIVEIRA, M. L. et al. Infecção por Chlamydia em pacientes com e sem lesões intra-epiteliais cervicais. Rev assoc med bras, v. 54, n. 6, p. 506-512, 2008.

RISSO, V. B. DA S.; SOARES, M. C. Avaliação dos registros do exame citopatológico em uma unidade básica de saúde em Rio Grande-RS. J nurs health, v. 1, n. 1, p. 84-90, 2011.

RODRIGUES, A. D.; BUSTAMANTE-TEIXEIRA, M. T. Mortalidade por câncer de mama e câncer de colo do útero em município de porte médio da Região Sudeste do Brasil, 1980-2006. Cad. Saúde Publica, v. 27, n. 2, p. 241-248, 2011.

SHIAVON, P. Avaliação da educação em saúde X Exame preventivo do colo do útero. Rio de Janeiro (RJ). 2009. Trabalho (Conclusão de Curso) - Pós-Graduação em Saúde da Família) - Faculdade de Medicina de Campos, 2009. Disponível em: <http:// www.fmc.br/cursos/posGraduacao/pdf/tcc18.pdf $>$. Acesso em: 20 nov. 2011.
SOARES, M. B. O.; SILVA, S. R. da. Análise de um programa municipal de prevenção do câncer cérvico-uterino. Rev bras enferm, v. 63 , n. 2 , p. 177-182, 2010.
Recebido em: 17/9/2015

Aceito em: 10/12/2015 